\title{
Photochemical Doping of Protonic Transistors from a Cephalopod Protein
}

David D. Ordinario, ${ }^{\dagger}$ Long Phan, ${ }^{\dagger}$ Yegor Van Dyke, ${ }^{\dagger}$ Tam Nguyen, ${ }^{\dagger}$ Amanda G. Smith, ${ }^{\dagger}$ Michael Nguyen, ${ }^{\dagger}$ Nikka M. Mofid, ${ }^{\ddagger}$ MyAnh Kaylee Dao ${ }^{\dagger}$ and Alon A. Gorodetsky ${ }^{\dagger},,{ }^{*}$

'Department of Chemical Engineering and Materials Science, University of California, Irvine, Irvine, CA 92697, USA

Department of Electrical Engineering and Computer Science, University of California, Irvine, Irvine, CA 92697, USA

${ }^{\S}$ Department of Chemistry, University of California, Irvine, Irvine, CA 92697, USA

*To whom correspondence may be addressed. Email: alon.gorodetsky@uci.edu 
A) Device Schematic

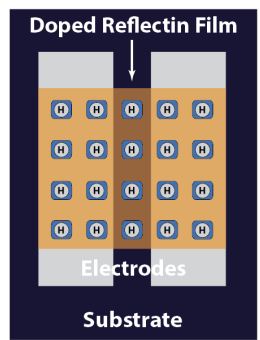

D) Device Schematic

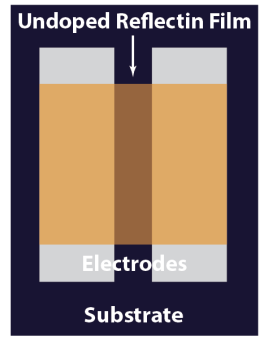

B) Optical Image

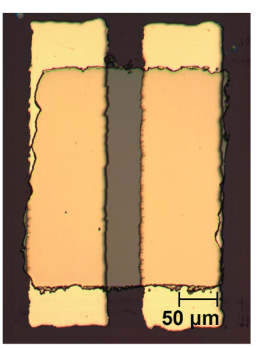

E) Optical Image

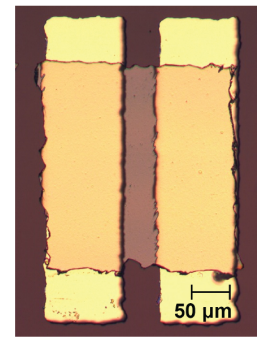

C) Fluorescence Image

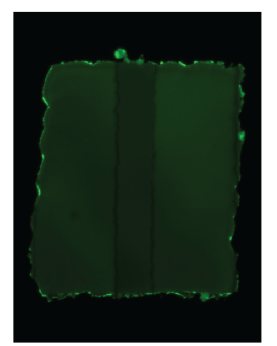

F) Fluorescence Image

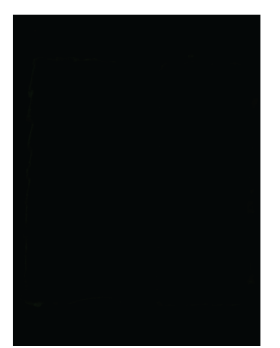

Supporting Information Figure S1. Microscopy images of device-integrated HPTS-doped and undoped reflectin films. A) A schematic of a two-terminal device where an HPTS-doped reflectin film bridges two electrodes. B) A typical optical microscopy image of a device-integrated HPTS-doped reflectin film. C) A typical fluorescence microscopy image of a device-integrated HPTS-doped reflectin film. D) A schematic of a two-terminal device where an undoped reflectin film bridges two electrodes. E) A typical optical microscopy image of a device-integrated undoped reflectin film. F) A typical fluorescence microscopy image of a device-integrated undoped reflectin film. 


\section{A) Without Blue Light With Blue Light}
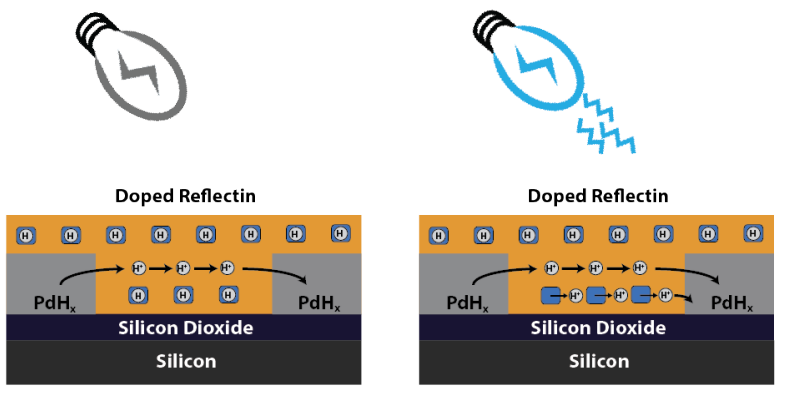

B)

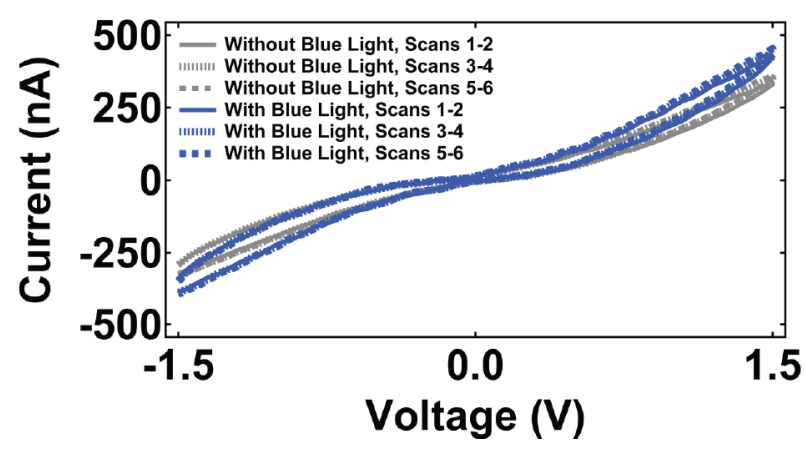

Supporting Information Figure S2. Demonstration of the stability of an HPTS-doped reflectin film during consecutive electrical scans. A) An illustration of a two-terminal HPTS-doped reflectin-based device without and with blue light illumination. Illumination of the device with blue light photoexcites HPTS and induces the release of protons into the film. B) The current versus voltage characteristics of a typical device from an HPTS-doped reflectin film without (gray) and with (blue) blue light illumination. Without blue light illumination, the electrical properties of the device show very little variability $(\leq \sim 3 \%)$ over 6 consecutive scans (the gray solid, dashed, and dotted lines are almost identical). With blue light illumination, the electrical properties of the device also show very little variability $(\leq \sim 3 \%)$ over an additional 6 consecutive scans (the blue solid, dashed, and dotted lines are almost identical). 


\section{A) Without Blue Light With Blue Light}
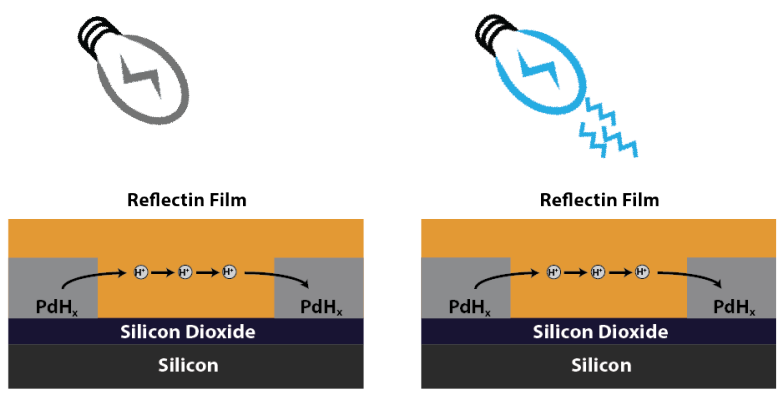

B)

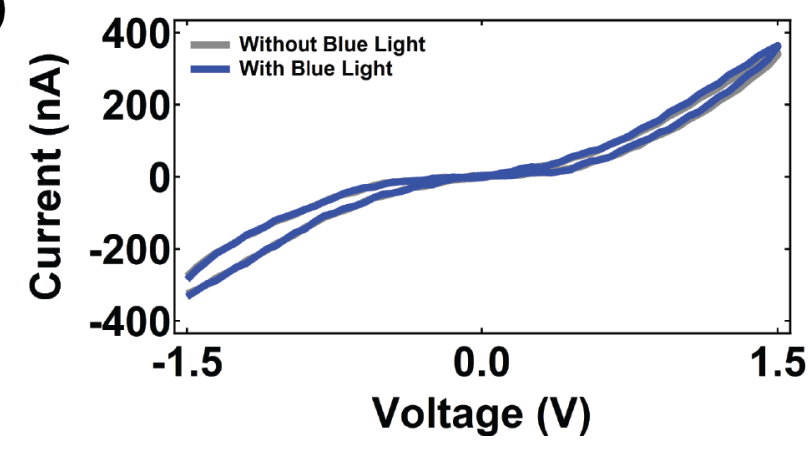

Supporting Information Figure S3. Electrical properties of an undoped reflectin film. A) An illustration of a two-terminal undoped reflectin-based device without and with blue light illumination. Illumination of the device with blue light has no effect on the charge carrier concentration in the film. B) The current versus voltage characteristics of a typical device from an undoped reflectin film without (gray) and with (blue) blue light illumination. Both the forward and reverse scans are shown. 


\section{A) Without Red Light}
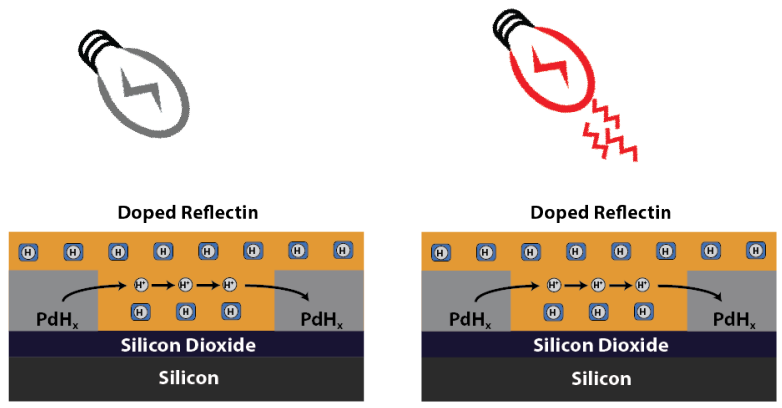

B)

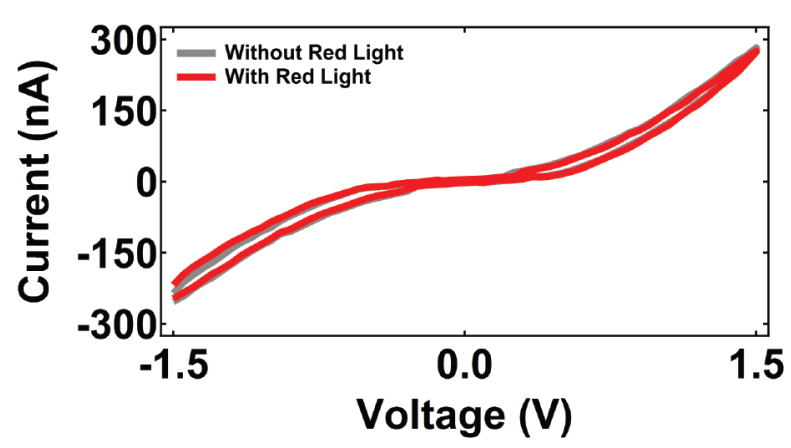

Supporting Information Figure S4. Electrical properties of an HPTS-doped reflectin film under red light. A) An illustration of a two-terminal HPTS-doped reflectin-based device without and with red light illumination. Illumination of the device with red light has no effect on the charge carrier concentration in the film. B) The current versus voltage characteristics of a typical device from an undoped reflectin film without (gray) and with (red) red light illumination. Both the forward and reverse scans are shown. 


\section{A) Without Blue Light}

\section{With Blue Light}
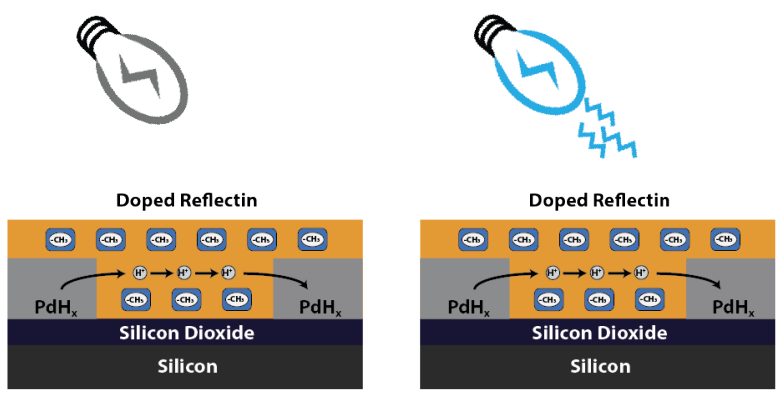

B)

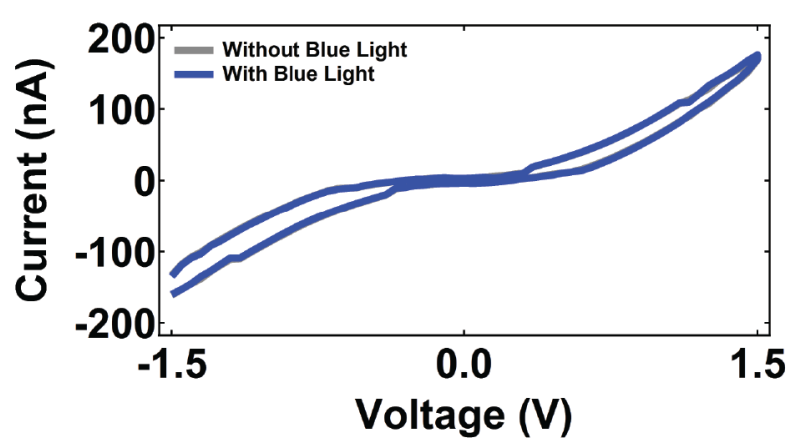

Supporting Information Figure S5. Electrical properties of a MPTS-doped reflectin film. A) An illustration of a two-terminal MPTS-doped reflectin-based device without and with blue light illumination. Illumination of the device with blue light has no effect on the charge carrier concentration in the film. B) The current versus voltage characteristics of a typical device from an MPTS-doped reflectin film without (gray) and with (blue) blue light illumination. Both the forward and reverse scans are shown. 


\section{A) Without Blue Light With Blue Light}
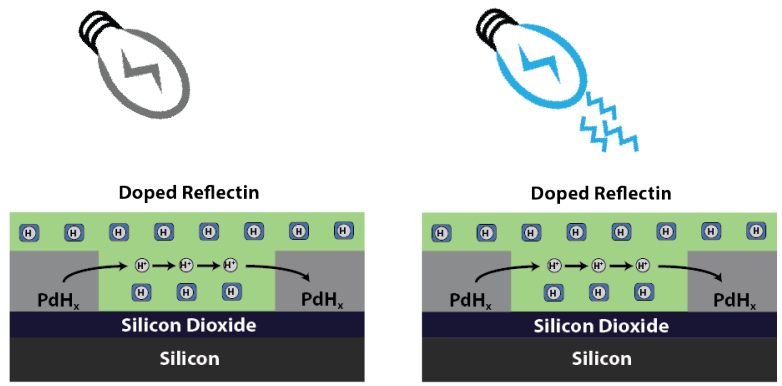

B)

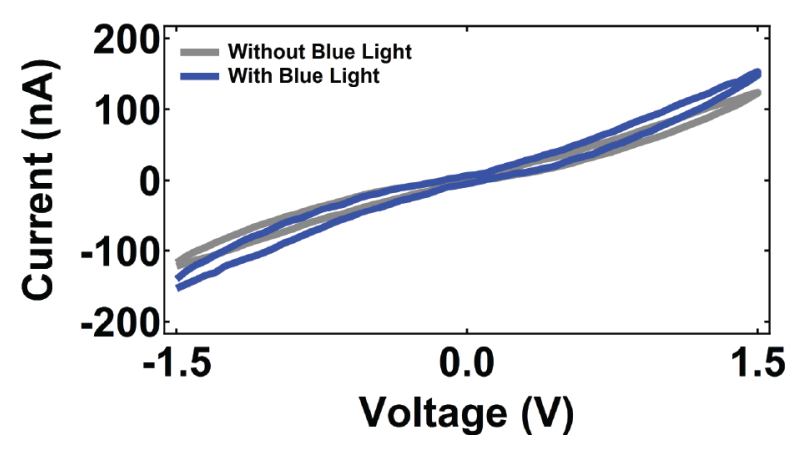

Supporting Information Figure S6. Electrical properties of an HPTS-doped reflectin mutant. A)

An illustration of a two-terminal HPTS-doped reflectin mutant-based device without and with blue light illumination. Illumination of the device with blue light photoexcites HPTS and induces the release of protons into the film. B) The current versus voltage characteristics of a typical device from an HPTS-doped reflectin mutant film without (gray) and with (blue) blue light illumination. Both the forward and reverse scans are shown. 